\title{
Powder Patterns from Nanocrystal Lattice Images
}

\author{
Eric Mandell $^{1}$, P. Fraundorf ${ }^{1}$, and M. F. Bertino ${ }^{2}$ \\ ${ }^{1}$ Dept. of Physics \& Astronomy and CME, University of Missouri-StL, St. Louis MO 63117 \\ ${ }^{2}$ Department of Physics, University of Missouri-Rolla, Rolla MO 65409
}

Fringes in electron phase, and Z, contrast images contain (among other things) the same "Fourier phase information" used in conventional darkfield imaging to locate objects of selected periodicity. However, conventional darkfield apertures often vignette in azimuth, and/or cannot distinguish frequencies whose spacing differs by less than $10 \%$. Lattice images of multiple nanocrystals facilitate an alternate approach to analyzing both fringe abundances (illustrated here), and cross-fringe correlations (discussed in a separate abstract).

At the University of Missouri - Rolla, AgPt nanoparticles with high aspect ratio [1] were grown in "necklace form" with crystal links 3-5 $\mathrm{nm}$ long and 2-4 $\mathrm{nm}$ in breadth, separated by occasional "large" equant knots (up to perhaps $10 \mathrm{~nm}$ in diameter). The question we address here: Can HREM imaging provide insight into segregation between Ag and Pt across the various components of these nanochains, given that $\mathrm{Ag}$ and Pt have strong (111) diffraction peaks at spatial frequencies of $2.36 \AA$ and $2.26 \AA$ respectively [2], making it impossible to distinguish them grain by grain e.g. by darkfield imaging?

Fig. 1 is a 240,000x magnified image taken with a Phillips EM430ST microscope, which shows the chain-knot strands streaming off of a large clump of silver. Using Adobe Photoshop with IPTK plugins and/or ImageJ, we are able to take power spectra of selected regions of specimen images digitized at $2400 \mathrm{dpi}$, and measure spatial frequencies (Figures 2 and 3). By taking many individual power spectra of various parts of chains and knots and measuring spatial frequencies, a histogram of spatial frequencies associated with links and knots was then compared to powder diffraction profiles of $\mathrm{Ag}$ and $\mathrm{Pt}$.

Fringe spacings thus allow one to correlate with each object type the range of randomly encountered Bragg spacings. Interesting patterns emerge from the data in Fig. 4. For example, the profiles in Fig. 4 suggest that the knots are largely Ag. Our analysis also suggests that the chains have a sharp peak in lattice parameter, quite near the $2.26 \AA$ peak for Pt though shifted slightly towards the higher lattice spacing of Ag. Projection broadening of fringes due to the small (e.g. $2 \mathrm{~nm})$ grain cross-sections is one possible explanation.

Specimens with less similar structures would of course make identification of trends even easier. Although the analysis described here was done by manually analyzing power spectrum peaks in 136 individual grains, the technique also lends itself to automation by simple azimuthal intensity averaging, provided regions for differential comparison can be otherwise flagged for the computer. Polycrystalline films in profile, with hopelessly overlapping grains, can also be analyzed in this way. The usefulness of this strategy will increase with the availability of large digitized images containing projected lattice fringe information.

References

[1] C. M. Doudna, M. F. Bertino, F. D. Blum, A. T. Tokuhiro, D. Lahiri-Dey, S. Chattopadhyay, and J. Terry, "Radiolytic synthesis of bimetallic Ag-Pt nanoparticles with high aspect ratio", $J$. Phys. Chem. B 107, 2966 (2003).

[2] R. G. W. Wyckoff, Crystal Structures (Malabar FL, 1982) p.7-10.

[3] J. C. Russ/Reindeer Games, The Image Processing Toolkit Version 3.0 (1999). 


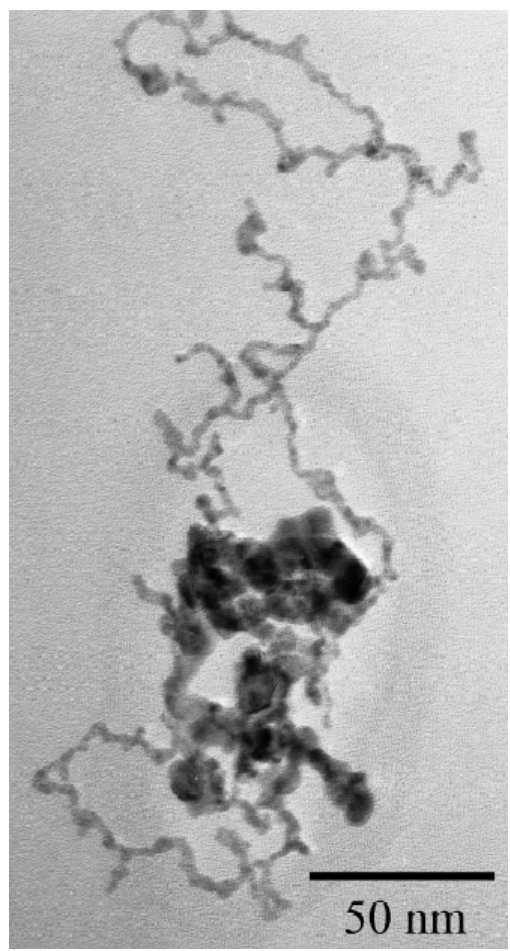

Figure 1

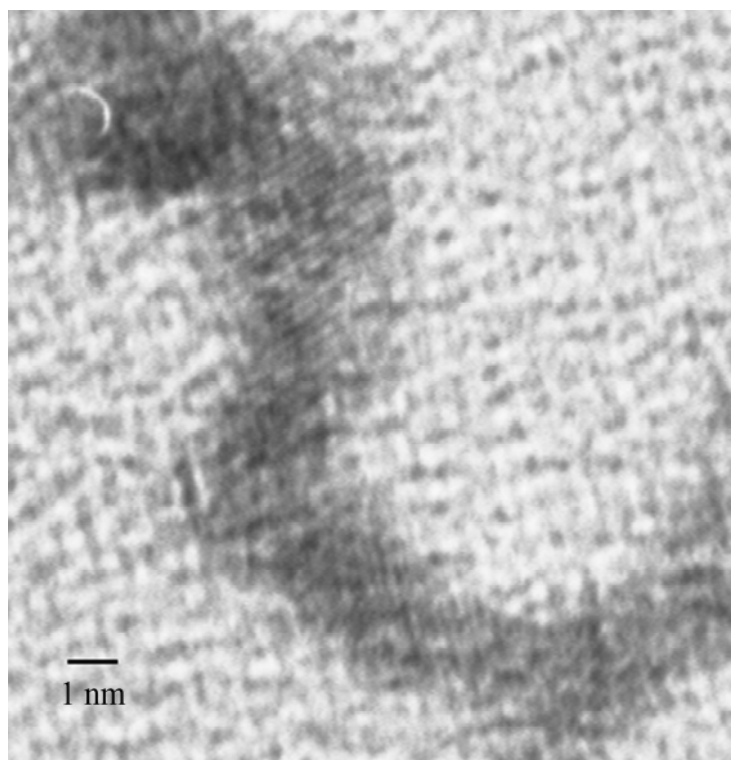

Figure 2
Observed Spacings

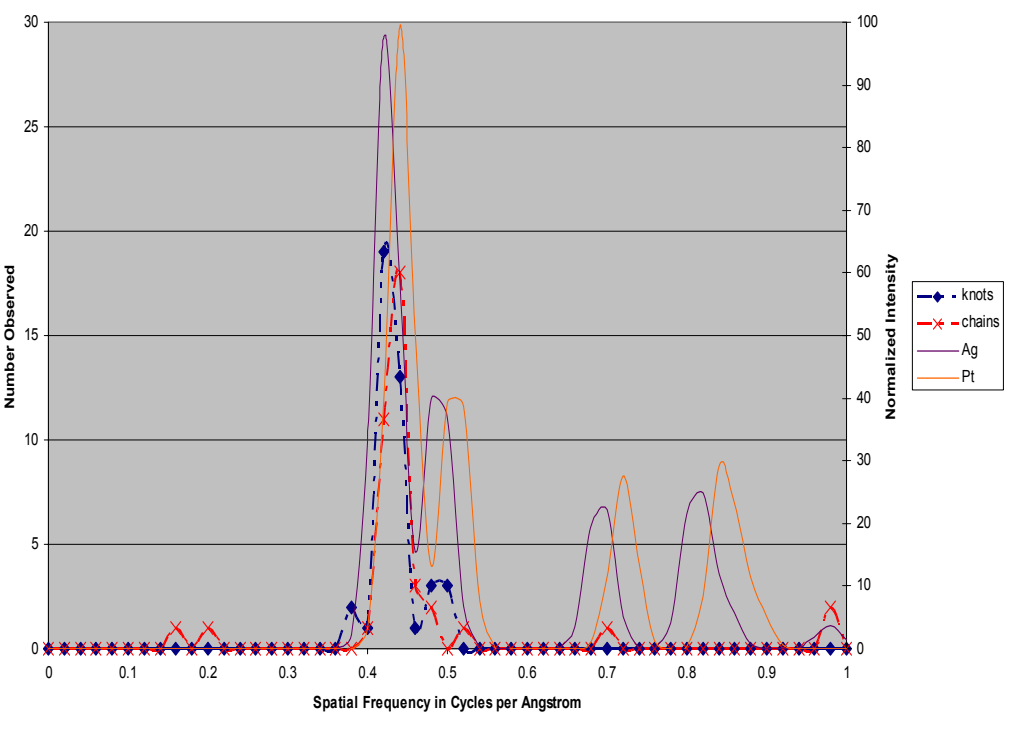

Figure 4

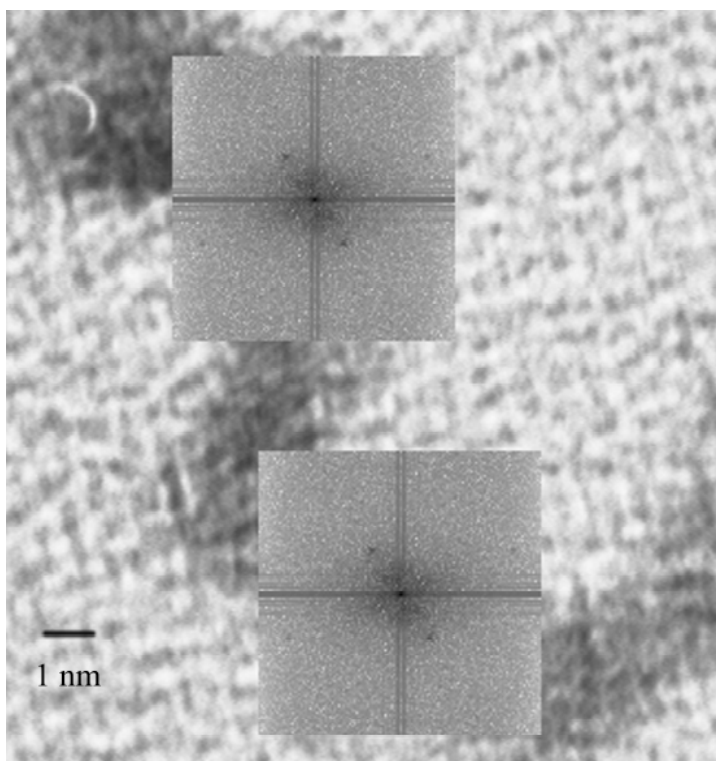

Figure 3

Figure 1: TEM image of AgPt nano-chains at 240,000x. Figures 2,3: Pt crystal "links" in a chain about $2 \mathrm{~nm}$ in width. The length to width ratio of the nano-crystals is approximately 3:2. Figure 4: A histogram comparison to diffraction data for Ag and Pt. The left-most solid line is Ag and the

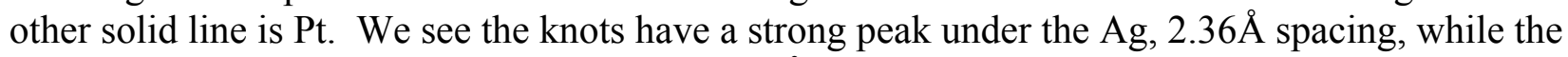
chains have a peak more centered under the $2.26 \AA$ spacing of $\mathrm{Pt}$. 